Editorial

\title{
Development of drug design for therapy
}

Keywords: chemical, salted water, drug design, chronic disease, antibiotics

\section{Editorial}

In our imaginary, for every kind of illness, there should exist a pill, whose immediate effect would heal our sorrow. The above is so true that in many cases a placebo, just a pill with no drug, would have some beneficial effect (and anyway the placebo is composed by something, even being sugar or magnesium, thus providing some chemical, not to mention the water usually used to take the pill, itself quite a healing chemical, being ourselves mainly salted water, even without thinking of the religious meaning of water). Of course this is not enough in most serious circumstances, nor for who is professionally or economically

engaged in drug design: our goal should instead be to identify the very origin of the illness (not just the symptoms when possible) and then design how to provide the proper chemical in order to bring back the steady health state where it was compromised, either by acute of even chronic disease, for which a permanent administration could be devised.

Such ability is not yet possible for every illness: this is way at least symptomatic drugs exist in order to at least minimize the effect of the symptoms when the underlying illness is anyway treated or at least healing in a different way: it would be dangerous to silence symptoms while not caring the real problem!

Even when the proper chemical is found and known, and favorably compared with possible competitors in terms of efficacy, adverse effects, cost, the problem is still how to administrate the proper dose at the very target place, without intoxicating too much the rest of the body while also wasting un useful chemical that in the very end will anyway pollute our world while not even creating resistance like antibiotics in bacterial. Administering such right amount directly at the very target in usually not even possible, and anyway not easy. The standard most tolerated, but at the same time less direct, way is to ingest a pill, who has to pass the digestive compartment before entering the blood, then diffuse to blood everywhere in the body to also arrive at target: pharmacokinetics and pharmacodynamics care to understand how much and how often to administrate in order to get the desired effect at target when the proper cover is found for the very molecule in order to arrive safe at target.

Parenteral administration does not look much different except that a different transfer compartment is used to reach blood in order to bypass the digestive one when the active principle would not stand it without being transformed in a not anymore useful way. Direct injection in blood do appear more easy for the drug, avoiding the

\author{
Volume 2 Issue 5 - 2018
}

\section{Diego Liberati}

Department of Bioengineering, Polytechnic University of Milan, Italy

Correspondence: Department of Bioengineering, Polytechnic University of Milan, Piazza Leonardo da Vinci 32, 20133 Milano, Italy, Tel +3934805693 I7, Email diego.liberati@polimi.it

Received: September 24, 2018 | Published: September 26, 2018

previous compartment, but is less comfortable for the ambulatory patient, needing a professional help for administration: the search of nano drugs is intended in improving such strategy, trying to embed the nano particle of drug in such a way that it could be chemically preserved by interactions while traveling in blood to target and maybe physically driven to target in a more controlled and fast and direct way, for instance through magnetism.

No matter of which of the recalled administration methods is chosen, but of course taking into account of the choice, the key necessary point is anyway to discover the very molecule, specific, more useful than already existing competitors and possibly less obnoxious in adverse effects. Several tool do exist nowadays besides the necessary intuition of the researcher. On one side machine learning help to predict characteristic of the new molecule with respect to important behavior, like for instance hydrophily versus hydrophobic behavior, while molecular simulation through systems of coupled Shroedinger equations for every single atom could help in understanding very subtle modification deriving by just few atoms substitution in a family of known molecules.

As often, no one approach is really always better than another, but the whole increasing and refining portfolio helps to better and better control the discovery process, helping the researcher, and more often the often interdisciplinary team, to better improve their already deep skills in the process.

\section{Acknowledgments}

None.

\section{Conflict of interest}

Author declares that there is no conflict of interest. 